\title{
Living with the Genome, by Angus Clark and Flo Ticehurst, within the Muslim Context
}

\author{
Ayman Shabana ${ }^{1}$
}

Modern genetics has ushered a new phase in human history and revolutionized human understanding of how living organisms are constituted and how they function. More particularly, it has revealed the processes associated with the transmission of inheritable features and characteristics to subsequent generations. The new knowledge that it generates brings promises of unprecedented preventive as well as therapeutic possibilities, especially as far as inherited diseases are concerned. These unprecedented possibilities are by no means limited to the fields of health care and life sciences as they touch many other aspects of our lives. However, as much as this genetic revolution has given rise to new exciting possibilities, it has also raised important ethical questions pertaining to the production and application of genetic knowledge. It is within this context of the double-edged nature of modern genetics that Living with the Genome: Ethical and Social Aspects of Human Genetics has to be placed. Although published in 2006, the book still provides a useful introduction to the range of ethical, legal, and social implications of modern genetic research and technology. It comprises 42 articles on a wide range of topics, which are drawn from the Encyclopedia of the Human Genome (one of the co-editors of the book, Angus Clarke, was also the editor of the "Ethics and Society" section of this reference work). The book is intended to enhance the readership of these topics by making these articles available to a wider audience beyond specialists in human genetics.

In terms of its basic subject matter, genetics aims to study how living organisms both change and maintain their basic characteristics over time. This

1 Associate Research Professor at Georgetown University's School of Foreign Service in Qatar (SFS-Q), as2432@georgetown.edu. This publication was made possible by NPRP grant \# NPRP8-1478-6-o53 from the Qatar National Research Fund (a member of Qatar Foundation). The statements made herein are solely the responsibility of the author.

(C) AYMAN SHABANA, 2019 | DOI:10.1163/9789004392137_011

This is an open access chapter distributed under the terms of the prevailing CC-BY-NC License at the time of publication. 
study is undertaken at three distinct levels: life of the cell, life of an individual organism, and history of the population of a particular species. Genome stands for the entire set of genes (of an individual organism or of an entire population) and genomics refers to the study of genes in this collective sense. Modern genetics traces its roots to important discoveries during the 19th century (Mendel's attribution of inheritance to certain particles, discovery of chromosomes, development of statistics and its application to hereditary processes, and Darwin's theory of evolution), which inspired further developments during the 2oth century (identification of DNA as the chemical basis of heredity and development of molecular biology). The past few decades have witnessed increased interest in the deployment of genetics in medical research and practice with the hope of identifying genes associated with particular diseases and developing effective ways not only to treat but also to prevent the occurrence of such diseases at the individual and collective (population) levels. Genetics is also used in the development of new drugs as well as their administration for certain diseases or even tailoring them for individual patients. Use of genetic examination in health care and medical practice is already replacing traditional physiological and biochemical methods and is expected to increase even further in the future. Important examples include confirmation of the diagnosis of certain diseases in individual patients and screening for certain disorders within particular groups or populations. Yet, as noted above, despite these remarkable therapeutic potentials of modern genetics, it raises a host of ethical, legal, and social concerns pertaining to proper use as well as implications of genetic information. The articles selected for inclusion in this volume explore these concerns with varying degrees of length and depth. They are divided into six main parts (prefaced by general editorial introductions): The Human Genome Project: Genetic Research and Commercialization; Genetic Disease: Implications for Individuals, families and Populations; Disability, Genetics and Eugenics; Genetics and Society: Information, Interpretation and Representation; Genetic Explanations: Understanding Origins and Outcomes; and Reproduction, Cloning and the Future.

The richly diverse collection of contributions that Living with the Genome contains offers only a glimpse of the range of concerns that biomedical technology has engendered. With the extremely fast pace of technical advances in this field, new discoveries or inventions spark new questions and launch new debates to examine their (bio)ethical implications. Still, this book remains a useful starting point as it captures some of the most important issues that remain as relevant now as they were when the book was published more than a decade ago. The main limitation of the book, however, is its limited scope of coverage as it focuses almost exclusively on the Western geographic as well as 
intellectual context, with very few exceptions (e.g. chapter on the Maternal and Infant Health Care Law in China, 147; brief references to discriminatory sex selection practices in India, 293; and Muslim ban on gamete donation, 276). This is somehow understandable because these discussions coincided with the rise of the early waves of genetic and reproductive technology in the West, particularly in the second half of the zoth century. But, considering the increasingly globalized nature of our world and the global influence of Western medicine, experts and practitioners worldwide are also joining these bioethical discussions. At the practical level, the availability of the latest applications of biomedical technology, especially to those who can afford it regardless where they happen to live, has also stirred similar debates over important bioethical concerns. The book, therefore, calls for comparative analysis of the various ethical, legal, and social issues that it highlights in order to reflect the diversity and richness of particular societies, cultures, and religious traditions. Such comparative analysis would also highlight important parallels and similarities in various social and cultural contexts. One interesting example that the book discusses is the case of the deCODE project in Iceland (56-63), which can provide important lessons for countries, especially those with small populations. The case highlights the critical role that regulators should play in ensuring compliance to ethical standards and proper conduct of genetic research. Another example is the successful use of genetic screening (premarital and prenatal) in Cyprus for inherited hemoglobin diseases (114-121).

Within the Muslim context, bioethical debates can be traced to two main factors: globalization of the medical curriculum (including bioethics), and the arrival of various applications of biomedical technology. Bioethical deliberations in the Muslim world, however, have drawn heavily on the Islamic normative tradition, particularly on the Islamic legal tradition. Researchers often point out that bioethical discourses in the Muslim world are dominated by the Islamic legal discourse, which is evident in the increasing volume of legal opinions (fatwas) on almost each of the issues addressed in this book. One of the main problems with these disparate fatwas is lack of a consist methodology for the examination of bioethical issues. The past few decades have witnessed serious efforts on the part of jurists and medical experts to provide systematic examination of bioethical issues with the goal of developing guidelines that should inform professional practice as well as national policies and legislation. These collaborative efforts have been facilitated by a number of national and transnational institutions as well as a number of academic and research centers. Although, for the most part, the development of a comprehensive Islamic bioethical framework remains work in progress and the general state of bioethics differs from one national context to another, empirical research shows 
that in order for any treatment of bioethical issues to be taken seriously, it has to engage this evolving body of Islamic normative literature. The bulk of this literature revolves, for the most part, around some key documents in the form of resolutions, decisions, or institutional fatwas, particularly ones that are issued by prominent national as well as transnational institutions. Below I give a summary of the main parts of this book together with brief comments in light of this Islamic normative literature.

The first part comprises eight articles offering a historical account of the Human Genome Project (HGP) with a particular focus on the debate over the commercialization of genetic information, which was one of the driving forces behind this project until its successful completion in 2003 (two and half years prior to the scheduled deadline in 2005). This debate was fueled by two competing visions for the project. The first was championed by the private sector, as represented by Celera Corporation, and the second was represented by an international consortium consisting of major public and state-sponsored research entities. While the first advocated patenting and monopolizing genome sequencing data as new inventions, the second insisted that genomic data should remain freely available as a non-commercial shared human resource for further research and development. Other contributions in this section provide various perspectives on the rationale, objectives, and implications of the HGP, which overall are not quite sanguine. Contrary to the usual hype emphasizing the miraculous achievements of genetic technology these contributions identify and highlight significant issues that tend to be glossed over due to the usual and unquestionable embracement of technology and its equation with progress. Chief concerns that run through these contributions include: commodification of genetic data, ownership and subsequent use of genetic material, distinction between a novel invention and mere discovery of nature; implications of the control of the human gene pool for future generations; obtaining informed consent in genetic research; and exaggerated expectations of/for gene therapy. At the global level these concerns reflect larger tensions between multinational capitalist interests on the one hand and rights of indigenous people and their claim over native resources on the other. In general, these concerns are also echoed in normative Islamic pronouncements. While these pronouncements praise the remarkable potentials of genomic research, they urge careful evaluation of any procedure involving intervention in, or manipulation of, the human genome. They also warn against any commercial exploitation or monopolization of genetic materials. These reserved sentiments are reiterated in several documents such as: the recommendations of a seminar organized by the Kuwait-based Islamic Organization for Medical Sciences (IOMS) in 1998 under the theme of "Genetics, Genetic Engineering, Human 
Genome, and Genetic Therapy: An Islamic Perspective," the resolution of the Islamic Fiqh Academy (IFA), affiliated with the Muslim World League in its 16th session that was held in Mecca in 2002, and the resolution of the International Islamic Fiqh Academy (IIFA), affiliated with the Organization of Islamic Cooperation in its 21st session that was held in Riyadh in 2013.

The second part of the book includes seven articles covering a number of ethical concerns associated with genetic counseling. The process aims to enhance understanding of a genetic condition and to explore possibilities to avoid or cope with such a condition. It is often pursued for either health risk or reproductive purposes. Although it focuses primarily on scientific explanation of a particular genetic condition, it may also address religious and metaphysical questions that patients and their families feel they must address. This is particularly important in multi-religious or multi-cultural contexts reflecting various meanings for universal experiences of illness and suffering. Considering the large scope of Islamic ethical-legal regulations, which cover various aspects of a person's life, including matters of health and illness, genetic counseling acquires added significance within the Muslim context. Practitioners need to develop familiarity with distinctive religious and cultural features that may create potential tension with mainstream (Western) bioethical standards. For example, genetic counseling is guided by three main ethical principles: autonomy of the individual or couple, right to full information, and utmost level of confidentiality (p. 115). Several studies, however, point out significant difficulties in the implementation of autonomy in non-Western contexts, including Muslim ones. For example, these studies show that Western emphasis on individual freedom might not be compatible with certain religious and cultural norms placing more emphasis on communitarian ethics. Moreover, such emphasis on autonomy may clash with particular attitudes concerning controversial issues such as abortion, euthanasia, or cremation.

One of the unique consequences of genetic testing has been increasing public awareness of genetic risk for, or susceptibility to, particular health conditions not only for the individual undertaking the test but also for close family members. This, in turn, raises important questions on whether/how information concerning risk for others could be communicated. Research shows that the perception of susceptibility to genetic diseases is dynamic and varies according to several factors such as family history, gender, age, or economic standards. Some studies even question the utility of genetic testing for certain conditions, especially when positive results may lead to a fatalistic attitude of resignation rather than proactive behavioral changes (p. 105). Medical practitioners, therefore, should be sensitive to the religious conceptualization of illness as a means for spiritual refinement. In Islam, for example, illness is 
seen as an opportunity for personal growth and also a way to gain reward in the hereafter. Delivery of effective genetic counseling would, therefore, require careful attention to contextual factors such as cultural background and religious attitudes towards certain medical conditions or procedures.

The third part consists of five articles discussing the relationship between disability and genetics. Each of these contributions addresses the issue from a particular perspective ranging from a historical investigation of problematic precedents, lingering traces of these precedents in contemporary practices, distinction between Western and non-Western outlooks on this issue, perception and implications from a human rights perspective, and examination of particular disability groups. Historical accounts of the contentious relationship between disability and genetics often start with the indelible eugenic practices during the 2oth century in Europe and the United States ranging from positive eugenics to enforced sterilization and even euthanasia. Some of the important traces of eugenics in contemporary research revolve around exploration of genetic explanation for criminal behavior as well as genetic screening especially in regions witnessing higher rates of genetic diseases due to inbreeding or lower rates of migration, where genes causing diseases tend to cluster over time. Within the Muslim context, the issue of consanguineous marriage has stirred extensive debates. Scriptural sources delineate certain prohibited degrees within close family relationships, which define the boundaries of incest. No categorical prohibition, however, is indicated beyond these prohibited degrees. Although it is generally discouraged, actual practice has always varied from one region to another depending on dominant cultural norms. The past few decades witnessed extensive efforts throughout Muslim-majority countries, with the help of international organizations, to raise public awareness about the significant genetic risks that consanguineous marriage involves, especially in places where it is commonly practiced. On the other hand, traces of positive eugenics could be found in the creation of sperm and ova banks, particularly ones obtained from individuals possessing desirable physical and cognitive traits (p. 144-145). Several Muslim countries have already established biobanks not only to encourage organ donation but also to facilitate the procurement of tissue samples for scientific research. A significant portion of Islamic bioethical discourses is dedicated to the elucidation of proper guidelines that should govern organ transplantation. However, in light of Islamic regulations pertaining to lineage and family relationships, these guidelines often include specific reservations and restrictions concerning transplantation of reproductive organs, gamete (sperm and ova) donation, and also milk banking.

The fourth part focuses on several social issues related to the employment of genetics in the creation of medical profiles. It consists of seven contributions 
dealing with topics including emergence of the gene as a significant cultural icon, perception of the interplay between genetic and environmental factors, confidentiality of genetic information, implications of genetic data for insurance purposes, and role of genetic factors in the confirmation of racial and cognitive stereotypes. Heightened media attention to the role of genetics in the definition of one's identity has transformed the gene into a powerful cultural symbol. The gene has increasingly been used as the locus of personhood and has almost acquired the sacred status attributed to the soul or other similar entities in different cultures. The concepts of the soul (nafs) and spirit (rühn) feature prominently in Islamic bioethical discourses, particularly on issues associated with beginning and end of human life. This is mainly due to the fact that Islamic scriptural sources define inception as well as end of human life in terms of the infusion or extraction of this metaphysical entity. Consecration of the role of genetics, therefore, raises questions concerning the continued relevance of the classical religious conceptualization of the soul or spirit as the primary factor to settle questions related to issues such as abortion or the new definition of death on the basis of brain (stem) function. On the other hand, some of the most important ethical concerns that modern genetics gave rise to are associated with boundaries of individual privacy and confidentiality of personal (genetic) information. The main ethical challenge in this regard remains how to reconcile concerns for individual privacy with others' right to have access to shared genetic information. These privacy and confidentiality concerns may have significant social and even economic implications such as one's ability to obtain affordable health insurance. This, in turn, raises questions of social justice as well as equitable distribution of social goods and services among members of the society.

The fifth part focuses on the general theme of genetic explanations particularly of behavioral traits. It consists of seven contributions dealing with the manner in which genes are used for explanatory purposes within the context of the HGP, evolutionary accounts of natural selection, and emergence of counter evolutionary accounts such as creationism and intelligent design, genetic reductionism and determinism, and reinforcement of racial and ethnic characteristics. Most of these contributions point out the limitation of exclusive reliance on genes for explanatory purposes and call instead for a more nuanced account for the interplay of genetic as well as environmental factors. From a religious perspective these discussions may also inspire new reflections on classical theological debates on divine destiny and human freedom. While notions of genetic determinism can be read as a modern extension to theological determinism, man's role in the creation and manipulation of environmental factors can be seen as a reflection of human agency and freedom. 
The final part, comprising eight contributions, is devoted to the interaction between genetics and modern reproductive technologies in terms of regulation, range of choices, feminist perspectives, particular procedures, distributive justice, and impact on the future. The reproductive revolution that modern biomedical technology has unleashed forces a reexamination of the regulatory aspects of parenthood, particularly within cultural contexts where genetic and social definitions of parenthood are not coextensive (p. 184-187). Countries vary widely with regard to the legislative model they adopt but any regulatory model that a country ends up choosing would depend on a number of contextual considerations and "nuances in tradition, religion, culture, economics, and wealth" (272). In general, while a liberal approach would issue from a permissibility presumption on the basis of fundamental rights and freedoms, a restrictive approach would be driven by concerns such as disrupting natural order and playing God. Ultimately, the range of possibilities that modern genetic and reproductive technologies generate would raise moral questions on fair distribution of benefits and burdens in society as well as on potential implications for future generations. In addition to these questions, ethical debates surrounding assisted reproductive technology (ART) within the Muslim context also address questions such as inter gender interaction in the clinical setting and also involvement of a third party in the procreative process. Islamic bioethical discourses often resort to classical ethical-legal concepts such as need (häjah), necessity (darürah), and utility (mașlahah) to evaluate particular cases and scenarios. In light of these concepts a distinction is often made between medical (therapeutic) and non-medical uses of these technologies. In the case of cloning, for example, a distinction is made between therapeutic cloning, which is perceived as potentially useful, and reproductive cloning, which is depicted as dangerous and harmful. A similar distinction is made also with regard to fetal sex selection, which is unanimously allowed for therapeutic purposes while being permitted for family balancing only in limited situations and on an individual basis. Anthropologists also point out different views throughout Muslim-majority countries, which are sometimes developed along sectarian lines. The most famous example is the Sunnì-Shī'i divide on gamete donation, which is opposed by most Sunnī scholars while being allowed by some Shīî scholars. This distinction is rooted in the conceptualization of gamete donation and its analogy with adultery. While critics equate the process with adultery, supporters limit the definition of adultery to actual physical contact.

In conclusion, as its title indicates, this book introduces some of the important challenges that modern genetics create, which are here to stay. People need to learn how to live and cope with them. A fuller exploration of the ethical 
and social aspects of human genetics, however, requires further comparative studies reflecting additional complexity and nuance associated with particular religious or cultural contexts.

\section{References}

Clark, Angus and Flo Ticehurst (eds.). 2006. Living with the Genome: Ethical and Social Aspects of Human Genetics. 327. New York: Palgrave Macmillan 